\title{
Vorstellung einer Zellkulturkammer zur elektrischen Stimulation von Spiralganglienzellen (SGZ)
}

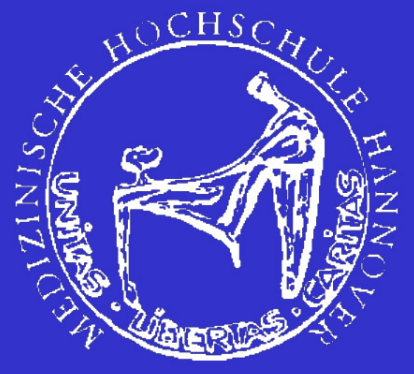

Hals-Nasen-Ohrenklinik der Medizinischen Hochschule Hannover

\section{Einleitung:}

In Folge von Hörminderung und Ertaubung kommt es nach Haarzellverlust auch zu einem Verlust der Spiralganglienzellen. Für den Erfolg von Cochlea-Implantat Operationen ist die Anzahl der erhaltenen Spiralganglienzellen entscheidend. Nach Tierversuchen mit ertaubten und einseitig mit einem Cochlea-Implantat versorgten Meerschweinchen (Kanzaki et al., 2002) konnte gezeigt werden, dass die Anzahl der überlebenden Spiralganglienzellen im Vergleich $\mathrm{zu}$ einer nicht implantierten Kontrollgruppe signifikant erhöht war. Diese Ergebnisse lassen die Schlußfolgerung $z u$, dass eine elektrische Stimulation und damit auch das CochleaImplantat an sich einen protektiven Effekt in Bezug auf das Spiralganglienzellüberleben hat. Die optimalen Stimulationsparameter hinsichtlich einer elektrischen Stimulation von Spiralganglienzellen sind bisher weitgehend unbekannt.

\section{Material und Methoden:}

Zur Untersuchung der Effekte einer elektrischen Stimulation auf kultivierte Spiralganglienzellen wurde eine Zellkulturkammer (Abb. 1) entwickelt, die es ermöglicht, Spiralganglienzellen unter definierten Bedingungen in einem Wechselfeld zu kultivieren. Nach einem Test der Kammer auf $\mathrm{pH}-$ und Temperatur-Konstanz des Zellkulturmediums unter Stimulationsbedingungen wurden initiale Versuche mit Fibroblasten (NHDF) durchgeführt. Die Stimulation der Zellen erfolgte bei konstanter Spannung über einen Zeitraum von $48 \mathrm{~h}$ mit Stimulationszügen von 50 biphasischen Pulsen $(10 \mathrm{~ms}, 50 \mathrm{~Hz})$ und einem InterburstInterval von 19 s. Nach Abschluß des Kultivierungszeitraums wurden die Zellen zurückgewonnen und mittels durchflußzytometrischer Analyse (Propidiumiodid-Färbung) auf mögliche Zellschädigungen untersucht. Zur Verifizierung der tatsächlichen Stimulationsbedingungen bei fester angelegter Spannung erfolgte eine Bestimmung der elektrischen Feldstärke in den Kultivierungskammern.

\section{Ergebnisse:}

Über den Stimulationszeitraum blieb der $\mathrm{pH}-\mathrm{Wert}$ des Zellkulturmediums unter den verschiedenen Testbedingungen konstant bei 7,4. Die Temperatur des Zellkulturmediums blieb bis zu einer angelegten Spannung von $40 \mathrm{~V}(480 \mathrm{~V} / \mathrm{m}$ in Kultivierungskammer, Abb. 3$)$ in einem akzeptablen Bereich für Zellkultivierungen. Die Ergebnisse der durchflußzytometrischen Untersuchungen zeigen bis zu einer angelegten Spannung von $40 \mathrm{~V}$ keine vermehrte Zellschädigung der Fibroblasten im Vergleich zur Kontrollgruppe (Abb. 4). Ab $50 \mathrm{~V}$ kommt es zu einem vermehrten Zelluntergang.

\section{Zusammenfassung:}

Die hier dargestellten Ergebnisse demonstrieren die Eignung der entwickelten Zellkulturkammer für Versuche zur Untersuchung der Effekte einer elektrischen Stimulation auf kultivierte Zellen, so daß das Setup jetzt einer Anwendung auf Spiralganglienzellen zugeführt werden kann.

Beschreibung der Zellkulturkammer:

- Zwei Elektrodenkammern (Abb. 2, A) mit Platin-//ridiumElektroden (B) und zentral gelegener Stimulationskammer (4 Chamber-Slide) (C)

- Quervernetzung zwischen Elektrodenkammern und Kultivierungskammern durch Glashohlbrücken (D) mit 1\% Agarose. Abdeckung durch Glasglocke (Abb.1)

- Befüllung der Kammern mit Zellkulturmedium, Platzierung in Brutschrank

- Reizapplikation durch Frequenzgenerator mit nachgeschaltetem Stimulusisolator (konstante Spannung)

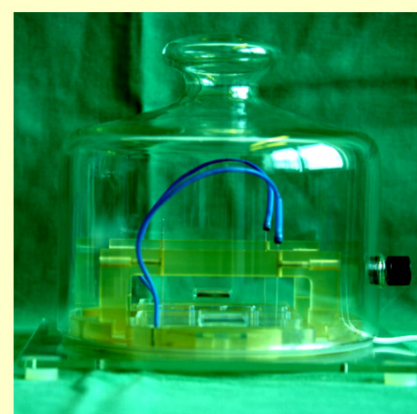

Abb. 1: Zellkulturkammer mit Glasglocke

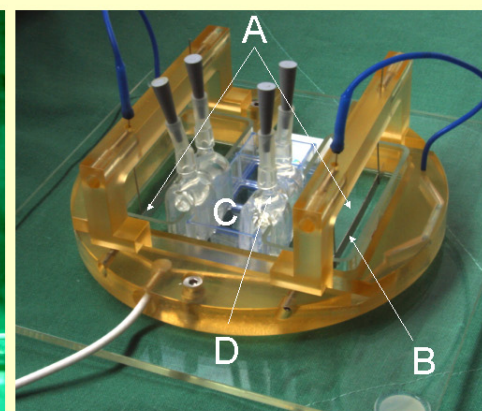

Abb. 2: Elektrodenkammern (A) mit Elektroden (B), Kultivierungskammer (C), Glasbrücken (D)

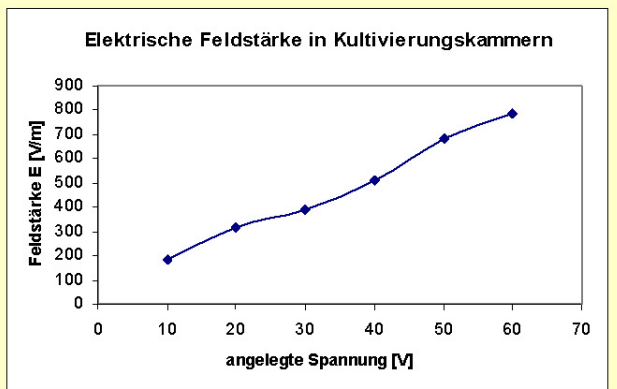

Abb. 3: Elektrische Feldstärke in Kultivierungskammern $(50 \mathrm{~Hz}, 1 \mathrm{~s}$ on, 19 s off)

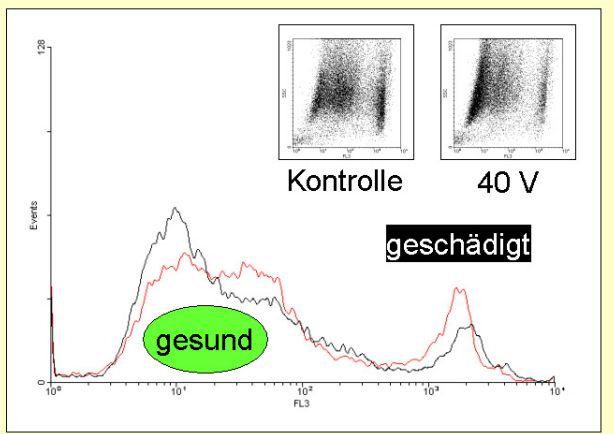

Abb. 4: FACS- Ergebnisse stimulierter und unstimulierter Fibroblasten. Gesteigertes Ausmaß von Zellmembranschäden korreliert mit verstärkterFluoreszenzintensität (x-Achse) 